\title{
El Negocio del Cable
}

\section{Rosa Maria Salom ${ }^{1}$}

\section{Resumen}

El desarrollo del sector de las comunicaciones se caracteriza por su dinamismo, por cambios en las formas organizativas (flexibilización y redes de organizaciones) y en la posición de los clientes. La vertiginosa velocidad de los cambios tecnológicos en este sector está afectando de manera dramática las estrategias clásicas de comercialización. La evolución del sector está provocando que las operadoras tradicionales se planteen estrategias en términos de sector mundial de las telecomunicaciones y no solo nacional. El presente estudio analiza las perspectivas de los teledifusores del Reino Unido frente al reto de la plena operatividad de la Unión Europea en 1999.

Palabras claves: Televisión, Cable, Telecomunicaciones, Estrategias, Negocio.

\section{The Cable Business}

\section{Abstract}

The development of the communication sector shows dynamism as well as clients changeableness. The enormous velocity of technological change in this sector is affecting dramatically the traditional market strategies. The evolution of this sector is pushing the traditional operators towards to strategies into international perspectives instead of national ones. This essay analyze the perspectives of the television broadcasting industry in the United Kingdom facing the full operation of the new European Union market in 1999. Key words: Television, Cable, Telecommunications, Strategies, Business.

\section{Recibido: 05-05-97 Aceptado: 26-06-97}

1 Licenciada en Comunicación Social. Master of Arts (Radio \& Television) San Francisco State University. Master in European Business Communication, Nottingham Trent University, Universidad Complutense de Madrid, Universidad Autónoma de Barcelona, Universidad de Siena. Profesora de la Escuela de Comunicación Social de la Universidad del Zulia (Pregrado y Postgrado). Ex-Jefe de Comunicaciones del Centro de Televisión Educativa de LUZ. Gerente-Fundadora de LUZ.FM 102.9. Especialista en Gerencia de Comunicación de Servicio Público y en Comunicación Corporativa.

Universidad del Zulla.. Escuela de Comunicación Soclal.

Avenida Ziruma, Maracaibo, Venezuela 


\section{Introducción}

En el Reíno Unido, la televisión pública muestra ingresos cercanos a los 1.641 millones de libras provenientes en un $95 \%$ de las licencias para hogares. Las inversiones por concepto de publicidad comercial alcanzan los 1.5 billones de libras por año, de los cuales 67 millones de libras corresponden a patrocinio. Para 1994, ITV permanecía como líder del mercado británico de la radiodifusión, seguido del Canal 4. Entretanto, la televisión vía satélite comienza a mostrar su potencial con beneficios de 170 millones de libras por año. (The European, 1995:17; Marketing, 1995:11; Snoddy, 1995:15; Kenyon, 1995:9; Irvine, 1994:14).

La industria de la televisión en el Reino Unido es, por to tanto, una industria productiva con beneficios que sobrepasan los 3 billones de libras al año. La gran interrogante es si la completa operatividad de la Unión Europea en 1999, creará nuevas oportunidades para los radiodifusores ingleses o una se constituirá en una amenaza. ¿Cuáles son las estrategias a seguir? Este estudio pretende formular algunas respuestas a estas interrogantes.

\section{El Mercado del Cable en el Reino Unido.}

Con un rango de 18 canales terrestres $^{1}$ y una oferta de más de 40 canales por cable y satélite ${ }^{2}$, el televidente del Reino Unido tiene una muy amplia variedad de oferta de canales a través de los cuales puede seleccionar un programa en particular. Además de esta oferta, los operadores anuncian el lanzamiento de nuevos canales: Playboy Channel, The History Channel, the Paramount Television Entertainment Network y el European Business News. (Financial Times, 1995:11)

Sin embargo, y a pesar de la casi total penetración de la televisión en los hogares del Reino Unido, el número de televidentes permanecerá igual. Por lo tanto, la competencia provocada por la aparición de nuevos canales a través de cable y satélite, unido a un uso cada vez mayor de la pantalla del televisor para otras actividades como los juegos y videos, sugieren que la audiencia de la televisión continuará declinando para los canales tradicionales, tal como lo mostró Music Television (MTV) recientemente a través de su propia investigación. (Hilk, 1995:27)Esta se constituye en una fuerte amenaza para la industria de la televisión en el Reino Unido. El primer impacto se

1 BBC1, BBC2, ITV y sus afiliadas: Anglia,Border, Central, Channel Grampian, Granada, HTV, HTV Wales, Meridian, Scottish, Tyne Tees, Ulster, Westcountry y Yorkshire y Channel 4.

2 Al momento de escribir este ensayo, la oferta consistia en: Sky One, Sky News, Sky Movies, Sky Movies Gold, The Movie Channel, The Disney Channel, Europost, Sky Sports, Sky Sports 2, The Christian Channel, The Sci-Fiction Channel, TLC, UK Gold, TCC, Nickeloden, Discovery, Bravo, Paramount, UK Living, Family Channel, MTV, VH-1, CMT Europe, Zee TV, Cartoon Network/TNT, Asianet, Travel, Channel One. Además de los servicios franceses: TF1 y Antenne2; el servicio italiano Raidue; el español TVE Internacional y Euronews. 
produjo con la introducción de ITV. En 1983, el Canal 4 y TV-am añadieron una nueva oferta al telespectador. Seis años más tarde, Rudolph Murdock lanzó sus canales Sky. Como resultado, la misma audiencia debió ser repartida entre más canales. Actualmente, los gerentes de ITV reciben fuertes criticas y reclamos de parte de los anunciantes, disgustados por la caida del rating. De hecho, el share de ITV bajó de $40,7 \%$ en 1994 a $36,3 \%$ en 1995. Debido a que los costos de tiempo en el aire permanecen estables, los anunciantes se quejan de que están forzados a pagar más por el mismo número de espectadores. Pero la división de la audiencia afecta a todos los canales. La creciente audiencia de la televisión vía satélite y el gran éxito del Canal 4 , causaron que la audiencia de la $B B C 1$ (el más importante canal de entretenimiento), bajará de $37 \%$ en 1990 a $32 \%$ en 1994 , mientras que la $B B C 2$ predice que al final de la década, la audiencia de la $B B C$ en su totalidad, caerá a un tercio del total de telespectadores. (Digman, 1995:4; Batmer, 1994:35)

2.2. Los mayores jugadores en la televisión del Reino Unido.

El panorama de la industria de la televisión del Reino Unido nos señala a la British Broadcasting Corporation (BBC) que opera tres canales de televisión: $B B C 1, B B C 2$ y $B B C$ World Service Televisión. La corporacicón controla cerca de cincuenta estaciones de radio: cinco nacionales, cuarenta locales y el World Radio Service. Mantiene cinco orquestas y publica revistas, incluyendo Radio Times,
Good Food, Wildlife, Clothes Showy Classical Music. El control de la corporación descansa en una junta Directiva designada por la Reina en consejo. Su presupuesto, cercano a los 1.7 billones de libras proviene de los impuestos a la televisión. (Balmer, 1995:34)

Rudolph Murdock es el principal accionista del Consorcio British Sky Broadcasting (BSkyB) que ofrece un paquete de canales internacionales, más servicios "premium". BSKyB es también el principal proveedor de las diez principales compañías de televisión por cable que operan en el Reino Unido. (Kenyon, 1995:7)

A pesar de nuestros esfuerzos no logramos identificar la composición del capital de ITV y del Canal 4. ITV y sus catorce contratantes regionales, especialmente cinco compañías de redes, además de producción independiente, son los productores de los programas que transmiten, mientras que la red está conformada, primariamente, por compañías pertenecientes a ITV. La selección de la programación local, recae en las manos de los contratistas regionales. Su principal fuente de ingresos es la venta de espacio para los anunciantes.

La producción independiente de programas del Canal 4, más algunos espacios importados, son la base para las exitosas ventas y subscripciones de las compañías ITV, las cuales venden espacio comercial al Canal 4.

El nuevo miembro del equipo es el canal 5, que se uni6 al grupo a finales del año pasado. La franquicia fue ganada por Channel 5 Broadcasting, un consorcio liderizado por Pearson, dueños del Finan- 
cial Times, Thames Television y Grundy Internationat; la compañía europea de medios $C L T$, que opera en Francia, Alemania, Belgica, Holanda y Luxemburgo y el Grupo MAl, que controla las compañías ITV Anglia y Meridian. Entre sus directores se encuentran Greg Dyke, antiguo gerente de TV-am y London Weekend Television; el otro socio es el banquero Warburg Pincus, que respaldó $L W T$ cuando Duke era Presidente. El consorcio ha anunciado que invertirá 110 millones de libras en programación por una audiencia correspondiente al $10 \%$ del share, 10 que significa una cobertura del $70 \%$ del pais (Bell, 199:20; The Daily Telegraph, 1995:4; Horsman, 1995:1)

Otra preocupación para los operadores es la aparición de otro jugador de peso pesado: la British Telecommunication (BT), lo que posiblemente dispare una guerra de tarifas. Actualmente a esta corporación se le ha prohibido su entrada en el sector de la teletransmisión de entretenimiento antes del año 2001, sin embargo, el partido Laborista ha señalado que podría permitírsele entrar al mercado antes de esa fecha. (Kenyon, 1995:14)

$Y$ se acaba de anunciar que durante la primera quincena del mes de junio de este año, el Gobierno Británico otorgó licencia a la British Digital Broadcasting (respaldada por el Granada Group y Carlton Communication) para operar una señal digital terrestre que ofrecerá 200 canales más interactividad. Al mismo tiempo, BSkyB ha anunciando que el próximo año pondrá en servicio una señal digital terrestre con 200 canales. Esto significará mayor competencia en el mercado británico. (Forrester, 1997)
Entretanto en los Estados Unidos, se calcula que el número de usuarios de cable modem crecerá de varios miles en la actualidad, a cerca de 4.5 millones para el año 2001. El estudio conducido por the Strategist Group, predice que los operadores de cable ofrecerán servicios de data a alta velocidad para 3 millones de hogares a finales de este año, y que esta cifra subirá a 20 millones de hogares para el cierre del 2001, el estudio incluso calcula que la penetración del cable modem alcanzará el $25 \%$ en cinco años. (Breznick, 1997)

En abril de este año, Microsoft compró Web TV Networks Inc., una empresa que permite al televidente accesar Internet (navegar a través del web y enviaro recibir correo electrónico a través de la pantalla del televisor). Esto seguramente acelerará los esfuerzos de la industria del cable norteamericano para poner el contenido de Internet en las pantallas de los televisores, al igual que los esfuerzos de los fabricantes de computadores personales para colocar la programación de TV en los PC. La compra, que alcanzó los 425 millones de dólares, transformará el acceso a Internet en un mercado de masas para fines de esta década. (Breznick, 1997)

MTV es uno de varios operadores de redes por cable que está trabajando con Intep Corp. para ofrecer a los usuarios de $\mathrm{PC}$, una nueva programación llamada Intercast. Este servicio, disponible para unos diez mil dueños de computadores, combina programas regulares de la TV con contenidos del Web. (Breznick, 1997). 
El panorama global del mercado del cable en el Reino Unido puede apreciarse en los gráficos No. 1 al 3 , los cuales reflejan la penetración de pantallas.

En este panorama, ¿cuál es el futuro más probable de las telecomunicaciones en el Reino Unido? Serios argumentos han sido sostenidos previamente por prominentes lideres que anticipan que la publicidad televisiva está en franco declive. Otros observadores, como Tony Kenton, Gerente General de IDK Media argumentan que en los próximos cinco años, la expansión de los servicios como el satélite y el cable no serán lo suficientemente grandes como para alterar realísticamente la economía de mercado. Por otra parte, otros como John Dimling, antiguo director de la Corporación de Servicio Público e investigador de la Asociación Nacional de Teledifusores, actual director de la Nielsen Media Research, plantea que "... considerando la velocidad y tecnología con la cual emerge la manera en la cual la televisión es transmitida, usada, comprada y vendida, planificar dos o tres años hacia el futuro es lo más lejos que nadie puede ir". (Dawson, 1995:26; Kenton, 1995:7; Barnatt, 1994:12)

\section{Del otro lado del Canal de la Mancha: Europa Occidental.}

Francia: El pais galo presenta una oferta de 3 cadenas por cable y tres ca. nales terrestres para el telespectador francés: TF1 (televisora pública, orientada comercialmente, líder del mercado); France 2 (canal público de orientación cultural) y la Cinq (de propiedad privada y orientación comercial)
El más nuevo de todos es La Chaine Info que comenzó transmisiones en Junio 1993 via cable. Este canal es propiedad de TF1. La Chaine Info es recibida en unos 500.000 hogares y se extenderá progresivamente a medida que más hogares posean cable. El canal produce beneficios cercanos a los 20 millones de francos.

Canal Plus, el principal proveedor de programas para el servicio francés de cable se ha convertido en menos de 10 años en el mayor éxito de televisión pagada de Europa, con más de 3.8 millones de subscriptores, un share del mercado global del $54.2 \%$ (muy adelante de $B S k y B$ ) y beneficios superiores a los 8.7 billones de francos, que deben estar actualmente sobre los 10 billones de francos. (Musnik, 1994:7)

El dinamismo de CanalPlus es ilustrado por sus conexiones con las tres principales cadenas de cable francés así como su diversificación en la producción de audiovisual y cine a través de sus subsidiarias: Ellipse Programme y the Studio Canal Plus. El canal produce dibujos animados, películas para televisión y películas francesas y en lengua extranjera. Desde 1990 sus facilidades han creado canales como $\mathrm{MCM}$, Eurosport y $\mathrm{Ca}$ nal $Y$, el Canal para Niños. Muchos de estos han sido exportados a otros países europeos, incluyendo el Reino Unido. $\mathrm{Ca}$ nal Plus siempre ha tenido ambiciones $y$ ha conseguido un exito total estableciendo sus formatos en Europa y África. Muy pronto se realizarás su lanzamiento en Turquía, Chile y Polonia. (Musnick, 1994:8) 
España: Desde la desregulación de la teledifusión española en 1990, se ha observado un rápido crecimiento de este mercado. La mayoría de los dueños de televisoras españolas vieron sus ganancias disminuir en 1993. La excepción fue Antena 3, la cual incrementó sus beneficios declarados de 1992 en un $78,4 \%$ y superó a sus rivales TVE (TVE 1 y TVE 2) propiedad del estado español, Tele 5 y FORTA (Federación de Organizaciones de Radio y Televisión Autónomas). Su éxito es el resultado de las grandes inversiones hechas en la programación y su decisión de concentrarse en mejorar sus bajas fuera del prime time. Ha contratado a las mejores personalidades de la televisión española, ha comprado los derechos para teledifundir películas nuevas y se ha dedicado a producir sus propias series. Sin embargo, TVE es el lider en el share de audiencia, lo que pudiera ser explicado por sus transmisiones deportivas. (Blanco, 1994:21)

Un aspecto particular del mercado de la televisión española es el relativamente importante rol cumplido por la publicidad no tradicional, como el patrocinio, la colocación de producto, competencia dentro de programas y promoción de programas, que representan el $49 \%$ del total de la publicidad. (Blanco, 1994:21)

En Septiembre de 1992, Murdoch y el gigante español de medios, el Grupo $Z$; con el apoyo del banco Banesto, compraron el $25 \%$ de Antena 3. Murdoch vendió posteriormente su parte, y Banesto busca nuevos compradores por estas acciones. Por otra parte, las acciones de Tele 5 se dividen entre el ex-primer ministro italiano, Silvio Berlusconi, $R T L$, Hachuel, el
Banco de Luxemburgo y La Once, quedando el resto de las acciones entre pequeños accionistas. Se ha comentado con insistencia que Berlusconi está tratando de vender su $25 \%$ de share. Varios inversionistas de Estados Unidos, Francia y Alemania aparentemente han mostrado interés. (Blanco, 1994:21)

Italla: La industria de la televisión en ese país tiene un nombre: Silvio Berlusconi. El ex-primer ministro es dueño del $90 \%$ de la televisión privada y del $60 \%$ del total de las televisoras. El total de la inversión publicitaria en Italia es de 2.9 billones de liras por año. (Taylor, 1994:17)

El sector duopólico se ha batido por la audiencia y los beneficios, con los tres canales de la AAI luchando contra Fininvest 3, Canale 5, Italia 1 y Retequattro. Ambos gigantes de la televisión se encuentran en rojo luego de varios años recortando las tarifas por publicidad y re. tándose en la programación. Se observa que la televisión por cable y vía satélite no son tan populares en Italia como en el resto de Europa Occidental:

El desarollo del cable es mínimo debido a que más del $30 \%$ de los italianos viven en pequeñas e inaccesibles aldeas y el cableado de sus hogares resultaria excesivamente costoso. En cuanto al satélite, hay una audiencia muy pequeña para los programas en idioma inglés. $E I$ canal nacional tiene los derechos para la transmisión de los más importantes eventos deportivos, por lo que el margen para canales como Europost es muy pequeño. Además, los canales terrestres ofrecen seis peliculas cada no* che. Para introducir el satélite hay que ofrecer algo más. (Taylor, 1994:17) 
El futuro de la televisión italiana luce inestable, desde que el presidente de la $R A /$ argumentó hace algún tiempo que la televisión de estado no debe competir con su principal rival, el grupo de canales Fininvest (propiedad de Berlusconi), sino que debe jugar un rol "complementario". Se han hecho llamados a los anunciantes para que disminuyan la publicidad y provean al público con un real servicio público; otros creen que el "canone", impuesto pagado por los televidentes, debe ser eliminado. Debemos esperar cambios dramáticos, puesto que el antiguo directorio de los llamados "profesores" -expertos sin afiliación política, han sido reemplazados por un directorio de "empresarios", muchos de los cuales han recibido el apoyo de al menos una sección del partido de la coalición de gobierno, Forza Italia. (Canepa, 1994:18)

Alemania: La escena mediática alemana no ha permanecido quieta y pareciera que permanecerá dinámica durante los próximos años. Hay 25 canales de televisión en el aire y al menos 3 más serán lanzados en breve. La noticia más dramática de los últimos años fue el fracaso, luego de sólo 15 meses en el nicho privado del canal Vox.

Vox fue lanzado en enero de 1993 como un pionero canal de "infortainment" (información y entretenimiento), centrado en la transmisión de programas de variedades y emisión de noticieros, pero el formato falló en calar. Las primeras mediciones dieron a Vox menos del $1 \%$ dei share de audiencia. Los anunciantes desertaron de la estación y las pérdida alcanzaron los 600 millones de marcos. (Meller, 1994:11)
Rudolph Murdoch compró el $49.9 \%$ de las acciones luego que Holzbrinck y Suddentsche Verlag se retiraron. Vox continúa en el aire y su programación ha sido cambiada a series de televisión, películas clásicas y deportes, con lo que su audiencia ha subido a un $2 \%$ del share.

Sorpresivamente, y a pesar de los problemas de Vox, al menos dos nuevos canales serán pronto lanzados al aire. EI canal de películas KabelKana/pondrá en el aire KabelKanal 2. A diferencia de los canales privado actualmente en el aire, KabelKanal será dirigido al mercado de la tercera edad (a partir de los 60 años). El segundo lanzamiento es el del canal de entretenimiento RTL Club, que competirá directamente con KabelKanal, y quizás con KabelKanal 2. Su llegada ampliará el Bertelsmann -CTL Networka tres estaciones, conjuntamente con la estación líder RTL TV y el canal RTL 2. Se espera que RTL Club se dirija a la audiencia de la tercera edad. En 1993, RTL TV se convirlió en la televisora privada que logró los mayores beneficios económicos $-130 \mathrm{mi}-$ llones de marcos, provenientes de los ingresos por concepto de publicidad que ascendieron a 2.5 billones de marcos. (Meller, 1994:11)

El otro protagonista importante de la pantalla alemana es Kirch -dueño de Sat 1 , canal que actualmente graves problemas de bja de audiencia, por lo que algunos directores de agencias de publicidad predicen que la estación se verá forzada a cerrar en breve sino cambia su programación y atrae más anunciantes.

Las estaciones públicas, ARD y $Z D F$, han anunciado que reducirán sus tarifas para publicidad en un esfuerzo 
para recuperar sus ingresos publicitarios, perdidos frente a las estaciones privadas. Luego de ver disminuir sus ingresos en 350 millones de marcos en 1993, ZDF ha rebajado sus tarifas en un $42 \%$. (Meller, 1994:12)

\section{Un recorrido por Escandinavia y Europa Oriental.}

Noruega: Este país tiene el mercado de televisión más dinámico de Europa en términos de crecimiento de share. El total de la inversión publicitaria en televisión en 1993 fue de 103 millones de dólares y se espera que continúe creciendo a una rata del $30 \%$ en los próximos años. (Edmunds, 1994:25)

$T V 2$, el primer canal comercial de televisión terrestre noruego tiene el $26 \%$ del share del mercado. Este canal subió sus tarifas para publicidad en un $25 \%$ en 1994. El espacio publicitario en TVNorge y $T V 3$, dos canales comerciales por cable noruegos, tienen vendido todo su espacio publicitario meses por adelantado.

Suecia: TV4 es el actual lider en la industria de la televisión sueca, con un $30 \%$ del share del mercado. El conglomerado mediático Kinnevik, lanzó en 1993 TV6, un canal cuyo espacio publicitario fue vendido tres meses antes de su lanzamiento. El espacio publicitario saltó en Suecia de 113 millones en 1992 a 186 millones el año siguiente. (Edmunds, 1994:25)

El mercado sueco se hará más fuerte, con una nueva legislación que promete nuevos canales comerciales nacionales y al menos 60 canales locales.
Finlandia: El primer comercial interactivo de este país fue presentado el año pasado en la pantalla del canal privado MTV3, como una colaboración entre Coca-Cola y la Telecom Finland.

Dinamarca: Tele Denmark firmó un joint-venture con Kinnevik y Televerket Norge para lanzar Nordic Satellite Distribution con diez y quince canales. Este país también recibe la señal de TVPlus, un canal dirigido por mujeres, propiedad del grupo Oslo-Schibstead. (Meller, 1994:25)

Polonia: Los publicistas polacos están preocupados debido a que el Gobierno lanzó un congelamiento de la televisora PolSat, para evitar su expansión, luego que ganó una licencia de transmisión nacional a comienzos de 1994. Los anunciantes cambiaron sus inversiones hacia el canal oficial TVP 1 porque este canal y la TVP 2, son las únicas televisoras con cobertura nacional. Sin embargo, estas estaciones tienen cuotas muy restrictivas de publicidad y les está prohibido cortar los programas con anuncios publicitarios. Los otros canales de televisión son Polania (privado), TV Polania (público), Canal + (privado) y Film Net (privado). (Meller, 1994:26)

República Checa: Un joint-venture polaco-norteamericano ha sido testigo del lanzamiento exitoso de Nova TV, canal comercial de cobertura nacional que ha obtenido paridad con los canales del Estado en términos de tamaño de audiencia y anunciantes. El tiempo de comerciales está limitado al $10 \%$ del total del tiempo de transmisión, comparado con el canal público $\mathrm{CT} 1$, el cual está restringido 
en su tiempo de transmisión de publicidad a un $1 \%$. (The European, 1995:13)

Hungría: Los publicistas están vigilando de cerca el crecimiento de la televisión húngara, donde la penetración de la televisión por cable y satélite ha alcanzado el $60 \%$ y nuevos canales comerciales, como TV3 están disfrutando de éxito. Por otra parte, la privatización de uno de los dos canales estatales está ocupando un lugar importante en la agenda de los políticos. (The European, 1995:17)

Luego de echar un vistazo a la situación de la industria de la teledifusión en el Reino Unido y de pasearnos por el panorama de la industria de la televisión en Europa, examinemos la más probables tendencias en la desregulación del mercado televisivo del Reino Unido y el inmediato futuro de la televisión.

\section{Flexibilización de servicios en la Televisión.}

Ha sido señalado que de una alta concentración de producción, y, por ende, un mejor uso de los equipos y personal de producción, una compañia se beneficiará de las economías de escala. Otro punto de vista es que la producción independiente es más eficiente. Sin embargo, en la producción de televisión no existen datos sistemáticos que nos permitan discriminar entre estas hipótesis. Los programas de televisión son, por naturaleza, heterogéneos, con grandes diferencias de calidad entre los tipos de programas, nuestra experiencia confirma las dificulta- des para calcular el costo promedio entre firmas productoras con diferentes volúmenes de producción, incluso con el mismo tipo de programas: La produción de Rafael Araujo, Lossada Girasol (mini-serie de ficción en video de tres capítulos de 25 minutos de duración cada uno), tuvo un costo total de 6 millones de bolivares; el documental-ficción en video (filmado en $16 \mathrm{~mm}$ b/n) de Ricardo Ball, Piragua del Sur (30 minutos de duración) tuvo un costo de 23 millones de bolivares ${ }^{3}$.

Por otra parte, el balance entre pequeños productores (generalmente llamados productores independientes) $y$ grandes compañías de producción pare. ce ser el resultado de condiciones reguladoras cambiantes por lo que no se pueden hacer inferencias de la data de firmas de diferente tamaño. Desde luego, la producción independiente sostiene a un gran número de empresas y crece a gran velocidad en la forma de empresas de servicios especializados de pre-producción, producción y post-producción.

En un artículo titulado "The emergence of flexible networks in the UK televisión industry", publicado por el British Journal of Management, $C$. Barnat y $L$. Stankey plantean que la industria de la televisión está "presenciando" la desintegración de las grandes organizaciones resultando en una creciente diferenciación en pequeños proveedores de servicios especializados y en el reemplazo de la rigidez de las relaciones, verticalmente integradas, con contratistas externos. (Balmer, 1994:232) 
Durante la última década, la industria de la televisión del Reino Unido se ha visto envuelta en un proceso de transformación estructural tanto en la salida de la teledifusión y los suplidores de nuevos medios al consumidor. Por to tanto, las viejas burocracias de alto costo han sido forzadas dentro de un nuevo modelo de operaciones, no sólo en la $B B C$ sino también en el sistema de ITV, para acercarse más al modelo de empresa flexible que depende cada vez más del uso de proveedores externos de servicios a todos los niveles.

Ya no es económicamente posible, incluso para grandes corporaciones, estar completamente equipadas en todos los formatos. Hay diferentes facilidades para la producción de televisión: 1 pulgada C, BVU, Betacam, una gran variedad de formatos de video digital, y película en 16035 milimetros, sólo para indicar algunos de los más comunes. La tecnología de producción es ahora más fácil de operar y confiable que en el pasado y es posible contratarla de multitud de ofertas. El costo es cada vez menos una barrera para entrar, tal como lo demuestran los canales de acceso público norteamericanos. (Balmer, 1994:232)

El rango de las potenciales facilidades de post-producción es también vasta, y la mezcla de formatos y equipos de post-producción que pueden ser integrados en una sola producción son ilimitadas. La selección de la adecuada tecnología de producción, o mejor, la mezcla de tecnologías, se ha hecho cada vez más importante en el altamente costoso y competido mercado de la producción independiente.
Una de las estrategias más distanciadoras dentro de la industria señalada previamente, tiene que ver con el uso de facilidades externas y la contratación de servicios, por ejemplo, los estudios contratados por día, equipos de producción alquilados por hora, revelado de película e impresión de copias facturadas de acuerdo al pie de película, etc. Las grandes compañías de televisión siempre han utilizado servicios propios en algunas áreas. Por ejemplo, cada vez más empresas contratan externamente el revelado y copiado de material cinematográfico, pues encuentran que las pequeñas compañías ofrecen ciertos servicios estandarizados a costos inferiores que sus propias facilidades in-house.

Esta flexibilización comienza a aparecer claramente en el mercado de la producción de televisión británica. Por ejemplo, en 1980, el lanzamiento del Canal 4, coincidió con el crecimiento de los productores independientes. En 1989, por ejemplo, Canal 4, comisionó programas a 526 productoras, 28 de las cuales, suplieron material por 1 millón de libras. El crecimiento de pequeñas compañías independientes dentro de la industria, muchas formadas sobre la base de una producción a la vez, significa que la "seguridad de permanencia" para el personal se está tornando escasa. (Balmer, 1994:232)

Estos cambios mayores en los procesos de producción no son exclusivos de ITV o de los productores de cable. Analizando la Identidad Corporativa de la BBC, J. Balmer, del departamento de Marketing de la Escuela de Negocios de la Universidad de Strathclyde, Glasgow, 
afirma que la $B B C$ debe ser mirada primero como un conglomerado. Por ejemplo, la $B B C$ comisiona programas; produce programas de radio y televisión; provee facilidades para la producción de programas; transmite programas; opera una agencia de noticias; provee servicios educativos; actúa como mecenas de las artes; desarrolla investigación técnica y social; se encarga de la publicación de revistas; distribuye programas a otras organizaciones de radiodifusión; actúa como recaudador de fondos para co-producciones y pone a disposición del público material grabado. Y concluye: "... la corporación muestra una ausencia de identidad clara". (Cavee, 1994:34)

El autor confirma que con la nueva Carta Real de 1996, se observarán cambios importantes en la identidad de la $B B C$, algunos de los cuales ya se han producido. Por ejemplo, El Acta de Radiodifusión de 1990 requiere que $25 \%$ de todos los programas de la $B B C$ sean realizados por productores independientes. Como consecuencia de esto, y como parte de la reducción general de costos de operación, mucho personal staff ha sido eliminado. Esto ha llevado a su fin la noción de que un empleo en la $B B C$ era un empleo de por vida. Durante 1993 la $B B C$ hizo programas maximizando eficiencia y efectividad de coslos al permitir a los productores escoger entre los servicios ofrecidos por la corporación y los ofertados por proveedores externos. (Cavee, 1994:34)

\section{Integración de redes en la industria televisiva.}

Una red es una organización que actúa como intermediario entre los productores de programas y los canales locales y que organiza la distribución de programas a más de un canal, tanto nacional como internacionalmente. Una cadena, puede significar la distribución de un sólo programa al menos a dos canales diferentes o puede proveer su programación hasta a doscientos canales distintos, como es el caso de algunas cadenas norteamericanas. La cadena puede estar organizada: a) por una combinación o cooperativa de canales locales, b) por una organización separada, c) por los compradores de programas mismos o d) por una combinación de los tres. (Campaign, 1995:11)

La distribución terrestre tradicional ofrece la posibilidad de economías de escala ( $a$ un costo marginal de 0 por espectadores adicionales) dentro del área de recepción; también ofrece un panorama de economía (disminución del costo por canal a medida que el número de canales aumenta) a través de la asociación para acceder a los servicios de satélite.

La distribución de programas via satélite ofrece un panorama de economia puesto que los costos fijos de lanzamiento del satélite se distribuyen entre más transpondedores; por añadidura, mientras más satélites se coloquen en órbita, menor será la capacidad de reserva re- 
querida y los riesgos de fallas del satélite pueden ser distribuidos entre todos.

En el caso de la distribución via cable, las economías de escala han sido estudiadas: el costo por canal, derivado de la construcción del sistema de cable declina a medida que el número de canales aumenta, especialmente debido a que el costo de tendido del cable, es virtualmente independiente del número de canales transportados. Las ventajas de costo de tamaño no se derivan primariamente de la distribución técnica de la cadena, sino por la habilidad de un operador grande para empaquetar y vender más efectivamente a subscriptores potenciales, básicos y compradores. (Campaign, 1995:11)

No existe una opinión unánime entre los dueños de media, anunciantes y especialistas. Por ejemplo, Graham Brown, Gerente General de Carat Televisión, una conocida compañía de media presente en toda Europa, inspirado por la adquisición de Capital Cities/ABC por 19 billones de dólares por la Corporación Disney, explica que fusiones como ésta, son familiares para los europeos, aunque en menor escala; recordemos los holdings de medios de Silvio Berlusconi y Ruldolph Murdoch. En sus propias palabras, Brown plantea sus ideas sobre el futuro del media "... Los dueños de media están liderizando la vía hacia el mercado global". Para lograr sus inversiones de capital, los dueños de media necesitan hallar economías de escala. Eso explica -dice él, por qué estamos viendo integraciones verticales. (Campaign, 1995:3)

La producción de programas podría estar repartida entre canales de teievisión en todo el mundo a partir de una sola compañía, lo cual requeriría una activa cadena internacional de media y una centralización de las ventas por los dueños de media. John Perris, Chairman de Zenith Media Worldwide (con una facturación de 2.8 billones de dólares en sus 21 oficinas en 14 países, incluyendo Europa Oriental) plantea que "Incluso donde las compañías alegan estar operando globalmente, mucha de la planificación y compra al detal es local. (Campaign, 1995:3)

Peter Mitchell, presidente de la Federación Mundial de Anunciantes y director de Asuntos Estratégicos de Guiness afirma: "Mientras los dueños de media han estado buscando economías de escala a través de la centralización editorial - trabajo de producción, han sido los anunciantes quienes han buscado la oportunidad para explotar la propiedad individual de títulos o canales en múltiples mercados. (Campaign, 1995:3-4)

¿Existe en el Reino Unido una auténtica marca global de televisión? En este momento la respuesta es no. La única cadena global en el mundo es $C N N$ y MTV. Entretanto, las cadenas compradoras de televisión continuan ampliando su cobertura de Europa, país por país, esperando que el anunciante se adapte al nuevo panorama de los media.

La actividad final a considerar es la generación de ganancias. Las principales vias financieras de los teledifusores son: 1) alguna forma de impuesto a la recepción; 2) el anunciante financia, es decir, las cadenas y los canales obtienen sus beneficios vendiendo espacios a los anunciantes y transmitiendo publicidad a lo largo de su programación la cual está 
libre de cargo para los telespectadores y 3) cargos directos, donde los subscriptores pagan una cuota para ver un programa o para accesar a un canal o paquete de canales durante un período de tiempo establecido. Hay obvias economías de escala en costos de venta y facturación: vender tiempo de publicidad nacional es más barato por hogar que venderlo localmente; facturar por canal mensualmente es más barato que facturar por programa.

En el mundo de la televisión hay oportunidades para la integración que han sido controversiales: las vinculaciones entre productores de programas y las cadenas, entre las cadenas y los dueños de canales locales y entre la administración de canales locales y el control de los sistema de distribución.

\section{Oportunidades para los teledifusores del Reino Unido.}

Hay diferentes alternativas para cubrir las amenazas de la desregularización del mercado: pérdida de mercados cautivos, eliminación de cuotas publicitarias, estandarización en el pago de impuesto por todas las televisoras, aparición de nuevos competidores tanto en la producción, como en la difusión de programas de televisión, etc. Por el momento las respuestas parecen ser:

\section{A.- Aumentar de tamaño de las cor-} poraciones de medios, lo cual es obstaculizado actualmente por parámetros legales. El Acta de Teledifusión de 1990 prohibe a las compañias de media del Reino Unido tener más del $20 \%$ de las acciones de otro medio nacional. Una compañia ITV sólo puede poseer un máximo del $20 \%$ accionario de un canal por satélite, un periódico nacional o una estación de radio nacional. Similarmente, una compañía de media con núcleo de negocios en periódicos está restringida de tener intereses y posesión en televisión terrestre. Los teledifusores terrestres existentes están limitados para poseer o controlar intereses en televisión por satélite o cable -pero la televisión por cable escapa de las restricciones de propiedad de más de dos licencias regionales para teledifusión. (Digman, 1995:19)

B.- Desarrollar una estrategia de nicho. El carácter distintivo de la televisión financiada por anunciantes es que los programas son ofrecidos "sin cargo" para la audiencia, mientras que las audiencias son vendidas como paquete a los anunciantes, de los cuales las estaciones obtienen sus ganancias. Una de las principales caracteristicas de la televisión soportada por publicidad, viene precisamente de la selección de programas: los cuales responden más al número de personas que lo observan que a sus méritos cualitativos. Un canal financiado por publicidad está interesado solamente en el número de personas que ven sus programas ( $y$ en alguna medida, se interesan en la composición de ese público), puesto que sus ganancias se derivan de la venta de espacio a los anunciantes.

En este escenario, la televisión comercial terrestre parece tener una relativa ventaja comparativa en la base de soporte de anunciantes, especialmente para la venta de productos de consumo masivo, porque su mayor audiencia será el principal atractivo para los anunciantes. Por ello, los anunciantes usualmente escogen canales terrestres gratis, de gran cobertura nacional, para promover la venta 
de productos y/o servicios de gran demanda como comunicaciones (teléfono, correo), finanzas (seguros, bancos), productos del hogar (detergentes, cosméticos, supermercados, alimentos, juguetes para niños y electrodomésticos), etc.

Para aquellos que escogen el nicho apropiado, los altamente especializados canales de cable parecen ser muy atractivos para vender productos sofisticados y/o especiales, debido al carácter de su audiencia. Los datos muestran que los docentes, profesionales, técnicos, artistas, intelectuales y en general, la gente con elevado nivel educativo tiende a favorecer los canales culturales tanto como aquellos de servicio público. Sin embargo, al observar la clasificación de la audiencia atendiendo a su condición socioeonómica, se observa que la clase mediaalta y alta, tiende a concentrarse en los canales por cable y satélite.

Por otra parte, las estaciones locales terrestres de provincia tienden a tener una audiencia más leal puesto que sus servicios tienden a favorecer los intereses locales más que consideraciones foráneas decididas en un algún misterioso sótano de la capital, o peor aún, del extranjero. Al mismo tiempo, los canales locales ofrecen noticias locales $y$ asuntos de servicio público, rara vez hallados en los canales nacionales. Esto puede ser también un enfoque para aquellos canales locales terrestres que tienden a tener un área geográfica grande bien definida. Para los negocios y servicios locales, ésta podría ser una vía interesante si el canal tiene éxito en mantener los costos bajos.

Una posibilidad adicional se eleva de los nuevos mercados abiertos en Eu- ropa, debido al incremento de la necesidad de programas. El creciente número de canales, tanto terrestres como por cable, reclamará con urgencia programas para alimentar las pantallas vacías. Mientras el costo por segundo de programación permanece alto, la economía de escala jugará un papel importante al vender programas a países que tienen el inglés como segunda lengua.

En el discurso del Chairman de Thames Televisión se informa que en 1992 las ventas de programas en el Reino Unido permanecieron fuertes en $81.8 \mathrm{mi}$ llones de libras y estos, conjuntamente con el aumento de ventas conjuntas de las cadenas en los Estados Unidos, ayudaron a incrementar la venta mundial de programas del Reino Unido a 125.6 millones de libras. Carlton Communication otra exitosa compañia inglesa de producción, anunció en 1995, luego de un giro de 879.312.000 libras en el mercado Europeo y del Reino Unido y ventas por 525.384.000 libras en el mercado norteamericano, "su intención es expandir esta fuerzas mayor en la televisión británica, produciendo programas populares de alta calidad para el Reino Unido y los mercados internacionales". La venta de programas de Central Independent Television PLC para 1992 mostró un beneficio de 333.188 libras,en el caso de Yorkshire Tyne Tess Television, sus ventas de programas para el período 1993-4 alcanzaron los 109.516.000 libras. (Extel Financial Limited, 1995:1-3)

EI Reino Unido tiene una larga y rica experiencia en la producción de video y películas para televisión. Series ganadoras de premios, actualmente en las pantallas 
inglesas, serán bienvenidas por telespectadores en Europa. A pesar de que las compañías previamente mencionadas han tenido éxito en los mercados de documentales y los programas populares de televisión, Granada TV y la $B B C$ parecen ser las más favorecidas por los recientes eventos de la Televisión Europea, puesto que ambas tienen la más antigua y exitosa experiencia en este campo.

Granada TV probablemente venderá sus ya probadas series, mientras que la $B B C$ ofrecerá sus documentales y tratará de introducir sus cursos de Inglés como Segunda Lengua, ampliamente conocidos en universidades y escuelas en todo el mundo y dentro del mercado PanEuropeo.

Otro servicio que puede ser proporcionado a este mercado es el doblaje de las series y programas de habla inglesa, las cuales podrian ser traducidas a los 30 idiomas oficiales de la Unión Europea y de los países de Europa Oriental.

Un tercer y creciente mercado es el segmento de la televisión educativa. Yorshire Tyne Tees Televisión está fuertemente involucrada en esta área, especialmente desde el arribo del CD-ROM. Esta compañía ha creado recientemente un joint-venture con Thomas Nelson, el brazo de publicaciones educativas de Thomson International, para desarrollar la producción y venta de programas en $C D$. ROM para establecimientos educativos, esta nueva aventura se encargara inclusive de los videos educativos de Yorkshire Tyne Tees. La participación de más compañias británicas de producción en este potenciaimente gigantesco mercado, es posible.

\section{Conclusiones}

La completa operatividad de la nueva Unión Europea en 1999 creará, desde nuestro punto de vista, nuevos mercados para la industria de la televisión del Reino Unido y fortalecerá otros.

La venta de videos y películas para televisión, parece ser el sector de mayor beneficio, debido a la gran disponibilidad de programas ya realizados para nuevos telespectadores, así como el alquiler de facilidades técnicas y de personal ya entrenado. Por lo tanto, las economías de escala parecen ser completamente aplicables. Sin embargo, no se debe menospreciar la respuesta de la industria de la televisión norteamericana, la cual sin duda responderá con movimientos en el área, de los cuales ya tienen fehacientes pruebas de electividad.

El sector del doblaje y la producción de software para video y medios audiovisuales (CD-ROM proveerá una prometedora área. Nuevamente, las mayores amenazas permanecen en la fuerte competencia de otros productores europeos, especialmente los franceses e italianos, quienes estấn mostrando alta eficiencia y productividad, tal como observamos en la Exposición Mundial de Multimedia de Cannes, Millia 96).

En algunos años, la completa desregulación de la industria televisiva del Reino Unido, mostrará un nuevo panora* ma para estos negocios. Sin embargo, en el futuro inmediato, la realidad técnica será diferente con la puesta en operaciones de la televisión de alta definición, la creciente popularidad de la televisión in* teractiva y la oferta de más de doscientos 
canales diferentes. Las nuevas oportunidades están todavía inexploradas.

\section{Referencias Bibliográficas}

Balmer, J. The BBC's corporate identity: Myth, paradox and reality. Journal of General Management. Vol. 19, No. 3, Spring 1994, pp. 33-49.

Barnatt, $C$. and Starkey, $K$. The emergence of flexible networks in the UK television industry, British Journal of Management, Vol 5, No. 4 (Dec. 94), pp. 251260.

Bell, E. No surprise, surprise in Channel 5 victory. The Observer 29 Oct.,

1995, p. 20.

Blanco, D. and Hazas J., Planners Take Quality Route. Campaign, 30 Sept., 1994, pp. 12-22.

Breznick, Alan, More Cable Modem Customers. Cable World, April 21, 1997.

\section{Tunning in Internet TV. Cable} World, April 14, 1997.

Canepa, M. Govt Puts Rai Under Microscope, Campalgn, 30 Sept., 1004, p. 18.

Campaign Report "Will Europe Follow Disney's lead?" 29 Sep., 1995, pp 3-6.

Cave, M. An Introduction to Television Economics, pp. 9-37. IN: HUGHES, G. and VINES D. Deregulation and the Future of commercial Television. Aberdeen University Press, 1989.

Clemens, J. Modelling the impact of satellite television, Admap, Vol. 26, No. 7 (Jul/Aug 91).

Cook, R. East meets Media. Campaign, 30 Sept., 1994, p. 23.

Curwen, P. High-definition Television. A Case Study of Industrial Policy versus the Market, European Business Review, Vol. 94, No. 1 (1994).

Dimling, J. How to measure the TV 'superhighway', Admap, Vol. 29, No. 2 (Feb. 94).
Dignam, C. Media industry reels over BBC expansions. Marketing, 2 Mar, 1995 , p. 9.

Dignam, C. ITV chastened by adland fury. Marketing, 15 Jun., 1995, p. 4.

Economisit "Take five" Vol. 335, No. 7913,6 May, 1995, pp. 29-30.

Economist "The BBC: Poor Auntie" Vol. 335, No. 7912,29 Apr, 1995 , p. 35.

Edmunds, M. Print Survives TV Onslaught. Campaign, 30 Sept. 1994, p. 25.

Extel Financial Limited, Various Report, 11 Oct., 1995.

Fiddick, $P$, Can the future be research, Campaign, No. 26 (Aug. 94).

Financial Times "Held back by lack of capacity". 7 Nov., 1995, p. 11.

Finn, W. Surplus staff, Personnel Today, Vol. 26. No. 8 (Feb. 93).

Fellows, J. Treasury issues in an independent TV company, The Treasurer, (May 94).

Forrester, Chris y Brophy, Lesley Cable Brushes Off U.K. Digital TV Winner. Cable World (Junio 27, 1997).

Frean, A. Legal threats as Channel 5 is won by second-highest bidder. The Times. $2 B$ Oct., 1995, p. 2.

Fry, A. Spreading across the small screen. Marketing, 18 May, 1995, pp. VIII-X.

Hewitt, M. C4 has to tackle the burden of prosperity. Marketing, 23 Feb., p. 11.

Horsman, $M$ and Williams, $R$. Losers fury at decision on channel 5 . Independent, 28 Oct., 1995, p. 1

Hulks, B. and Baker, K. BARB's new trontier. Admap, Vol. 30, No. 7, Jul-Aug. 1995, pp. 27-31.

Hughes, G. and Vines, D. Deregulation and the Future of Commercial Television. Aberdeen University Press 1989.

Irvine, S. Overreach for the Sky?. Euromoney, No. 308, Dec., 1994, p. 14. 
Kellaway, L. Business airs a new image (company TV), Financial Times, No. 3 (Sep. 93).

Kenyon, T. Temperatures rising for TV. Marketing, 20 Apr., 1995, p. 7.

Marketing "Loot sponsors swap-on-air TV programme" 20 Apr., 1995, p. 3.

Marketing "Sky takes ads to the cinema after ITV ban" 30 Mar, 1995, p. 3.

Marketing "Media watch: Channel 4" $16 \mathrm{Mar}$, 1995, p. 11.

Marquis, S. Hostility towards ITV must not hide its inherent value. Marketing, $9 \mathrm{Mar}$, 1995, p. 23.

Meller P., West Moves In. Campaign, 30 Sept., 1994, p. 26.

Miles, B. Management appraisal at Yorkshire Television, Executive Development, Vol. 4, No. 3 (91).

Mistry, T. The discreet commercialism of the BBC, Campaign, No. 9 (Dec. 94).

Muirhead, A. New lessons from old data, Admap, Vol. 30, No. 9 (Sep. 95).

Musnik, I., Media Owners defy red tape. Campaing, 30 Sept., 1994, pp. 7.8
Musnik, I; Dickenson, N; Taylor, T; Blanco, D; Hazas, J.; Edmunds, $M$ and Meller, $P_{n,}$ European media, Campaign, 30 Sep. 1994.

Nolan, D. Digital TV: the challenge for advertising, Admap, Vol. 29, No. 2 (Feb. 94).

O'Neill, S. Highest Bidder fails to land fifth channel. The Daily Telegraph, 28 October, 1995, p. 4

Perry, J. Measuring Change. Admap, Vol. 30, No. 2, Feb. 1995, pp. 14-16.

Phillips, W. Rupert's (Murdoch) rocky road, Admap, Vol. 27, No. 4 (Apr. 92).

Snoddy, R. Battle for Channel 5 won by MAI and Pearson. Financial Times. 28 Oct., 1995, p. 1

Snoddy, $\mathrm{R}$. Why less TV time is more precious for advertisers. Marketing, 15 Jun., 1995 p. 5

Taylor, T. Political Web Bewilders TV. Campaign, 30 Sept., 1994, pp. 17-18.

The Daily Telegraph "Party-poopers celebrate the bare fax of victory" 28 Oct, 1995, p. 4

Watkins, S. The watchdogs, Campaign, No. 6 (May 94).

Wade, G. Subscription television, Audio Visual, No. 221,(Apr. 90).

Gráfico No. 1

Penetración de Pantallas en el Reino Unido

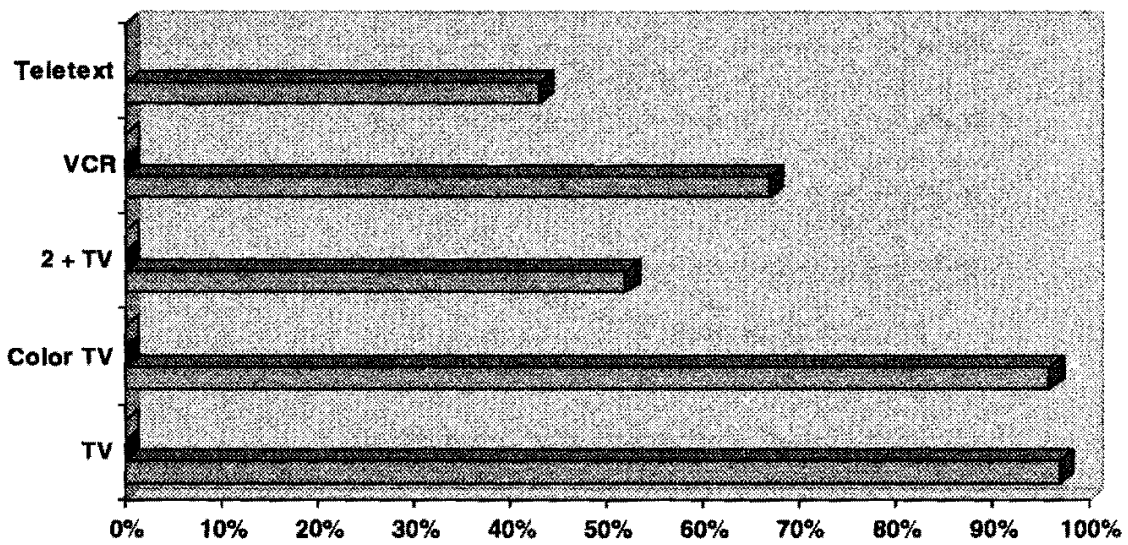

Fuente: The European Marketing Pocket Book 1994. 
Gráfico No. 2

Operadores de Cable líderes en el Reino Unido

$\begin{array}{lc}\text { Operador } & N^{\mathrm{a}} \text { de Hogares (000s) } \\ \text { 1.- Telewest } & 2.525 \\ \text { 2.- Nynex } & 2.509 \\ \text { 3.- Bell Cablemedia } & 2.023 \\ \text { 4.- SBC CableComms } & 1.258 \\ \text { 5.- Cable Tel } & 1.197 \\ \text { 6.- General Cable } & 739 \\ \text { 7.- Comcast } & 724 \\ \text { 8.- Videotronx } & 693 \\ \text { 9.- Telecential } & 613 \\ \text { 10.- Singapore } & 573\end{array}$

Fuente: Cable Communication Association, 1995.

Gráfico No. 3

Estimación de penetración de servicios de pantalla en el Reino Unido

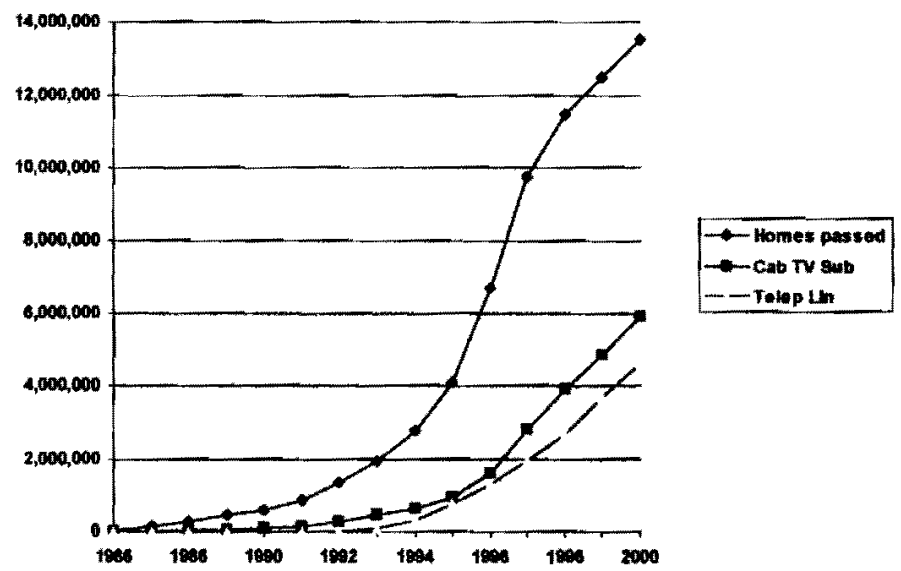

Fuente: The British CableCommunications Association. 